\title{
$\Omega$
}

Stefano Aliberti®, Amy Farr, Nathalie Tabin, Francesco Blasi,

Antoni Torres, Mark Woodhead, Giovanni Battista Migliori@,

Giovanni Sotgiu(1), George Dimopoulos, James D. Chalmers,

@ERStalk

Felix C. Ringshausen, Michael R. Loebinger, Robert Read, Gernot Rohde

D

For a full list of the authors' affiliations please see the Acknowledgements section.

\section{ERS syllabus for postgraduate training in respiratory infections: a guide for comprehensive training}

\section{Introduction}

Respiratory infections, including tuberculosis, represent one of the leading causes of morbidity and mortality across the world. They represent the deadliest communicable diseases causing 3.0 million deaths worldwide in 2016 [1]. The burden of respiratory infections can be unbearable for some health systems: they represent one of the most common reasons for doctor visits, regardless of age and sex [2].

Although respiratory infections have been identified as a mandatory topic in the education and training of respiratory physicians, the specialty has been faced with several challenges to implement training. There are ongoing discussions surrounding what is included and excluded epidemiologically within the parameters of respiratory infections. Thus it is no surprise that, at present, the vast majority of European countries do not yet have a formal system for educating respiratory physicians, at a specialty level, on the knowledge and skills considered essential in the diagnosis, treatment and prevention of respiratory infections. Furthermore, European countries have very different resources dedicated to the continuing development of respiratory professionals.

Keeping these educational and training challenges in mind, the European Respiratory Society (ERS) decided to support a group of experts in respiratory infections to define the core knowledge and skills considered essential to manage respiratory infections. The ERS respiratory infections educational task force was founded in 2014 and included 13 experts from six European countries (Italy, Germany, UK, the Netherlands, Spain and Greece). The task force had two main aims: 1) to develop a syllabus to guide the national training and education of respiratory physicians in the field of respiratory infections, and 2) to help structure ERS educational activities on respiratory infections.
Cite as: Aliberti S, Farr A, Tabin N, et al. ERS syllabus for postgraduate training in respiratory infections: a guide for comprehensive training. Breathe 2018; 14: 269-275. 
Table 1 ERS syllabus for postgraduate training in respiratory infections

\section{Module 1. Pathogens (respiratory microbiology)}

1 Respiratory microbiology

1.1 Normal bacterial flora

1.2 Classification of respiratory microorganisms

2 Respiratory bacteria

Mandatory

2.1 Gram positive

2.2 Gram negative

2.3 Atypicals

3 Respiratory viruses

Mandatory

3.1 RNA viruses (seasonal and/or pandemic, emerging)

3.2 DNA viruses (seasonal and/or pandemic, emerging)

4 Respiratory fungi

Mandatory

4.1 Candida spp.

4.2 Aspergillus spp.

4.3 Pneumocystis

4.4 Other fungi (including Mucor spp., Cryptococcus neoformans, Histoplasma capsulatum, Coccidioides immitis, Blastomyces dermatitidis, Paracoccidioides brasiliensis, Exophiala and Scedosporium)

5 Mycobacteria

Optional

Mandatory

5.1 Mycobacterium tuberculosis

5.2 Non-tuberculous mycobacteria (NTM)

6 Antimicrobial resistance

6.1 Mechanisms of antibiotic resistance

6.2 Multidrug-resistant (MDR) bacteria

6.3 Risk factors for MDR bacteria

6.4 MDR/extensively drug-resistant (XDR) tuberculosis

6.5 Risk factors for MDR/XDR tuberculosis

6.6 NTM resistance

6.7 Resistance in other microorganisms (anti-fungal and anti-viral resistance)

Optional

6.8 Influenza resistance

Optional

7 Microbiome

\section{Module 2. Host respiratory defence mechanisms against infection}

1 Natural barriers

Mandatory

1.1 Cilia/primary ciliary dyskinesia

2 Innate immune systems

Mandatory

2.1 Complement

2.2 Cells

2.3 Defensins

2.4 Cytokines

2.5 Inflammation process

3 Adaptive immune systems

Mandatory

3.1 Humoral immunity

3.2 Cellular immunity

4 Immune reconstitution inflammatory syndrome (IRIS)

Optional 
Table 1 Continued

\section{Module 2. Host respiratory defence mechanisms against infection (cont.)}

5 Pathophysiology of respiratory infections

Mandatory

5.1 Transmission

5.2 Infection

5.3 Inflammation

5.4 Resolution

\section{Module 3. Epidemiology, burden of disease and risk factors}

1 Epidemiological burden of respiratory infections

Mandatory

1.2 Bacterial infections

1.3 Viral infections

1.4 Fungal infections

1.5 Mycobacterial infections (tuberculosis and NTM)

2 Risk factors for respiratory infections and transmission

Mandatory

2.1 Environmental risk factors

2.2 Host risk factors (including other conditions leading to mild immunosuppression, e.g. diabetes mellitus)

2.3 Microbial risk factors

\section{Module 4. Diagnostic techniques for respiratory infections}

1 Indication and collection of biological specimens

Mandatory

1.1 Noninvasive (blood, urine, bronchoalveolar lavage, throat swabs)

1.2 Invasive (sputum, tracheal aspirate, thoracentesis, imaging-guided biopsy, transthoracic fine-needle biopsy)

2 Microscopy

3 Culture

4 Susceptibility testing

5 Immunological tests

Optional

Optional

Optional

Optional

5.1 Interferon- $\gamma$ release assay (IGRA)

5.2 Tuberculin skin test

5.3 Serology

6 Molecular testing

Optional

7 Rapid point-of-care diagnostic tests for viral and bacterial respiratory tract infections

Optional

8 Imaging techniques in relation to infections (including chest radiography, computed tomography, lung

Mandatory ultrasounds and magnetic resonance imaging)

\section{Module 5. General principles of antimicrobial therapy}

1 Antibacterial agents

1.1 Classification and activity (including pharmacokinetics/pharmacodynamics (PK/PD) principles)

2 Antiviral agents

Mandatory

2.1 Classification and activity (including PK/PD principles)

3 Antifungal agents

Mandatory

Mandatory

3.1 Classification and activity (including PK/PD principles)

4 Antimycobacterial agents

4.1 Classification and activity (including PK/PD principles)

5 Drug delivery or administration

Mandatory

5.1 Oral

5.2 Inhaled

5.3 Intravenous

5.4 Intramuscular 
Table 1 Continued

\section{Module 5. General principles of antimicrobial therapy (cont.)}

6 Drug-drug interaction

7 Antimicrobial adverse events

Mandatory

7.1 Haematological side-effects

7.2 Nausea and vomiting

7.3 Diarrhoea including Clostridium difficile infection

7.4 Ototoxicity

7.5 Hepatic toxicity

7.6 Nephrotoxicity

7.7 Cardiovascular toxicity

8 Principles of antimicrobial stewardship (including prevention of infection, infection control, adequate and appropriate treatment)

Mandatory

\section{Module 6. Common respiratory tract syndromes}

1 Common upper respiratory tract syndromes (including acute infective rhinitis, sinusitis, pharyngitis, epiglottitis, Mandatory laryngotracheitis)

2 Acute bronchitis

Mandatory

3 Bronchiolitis

Mandatory

4 Exacerbation of asthma

Mandatory

5 Exacerbation of chronic obstructive pulmonary disease (COPD)

Mandatory

6 Exacerbation of bronchiectasis

Mandatory

7 Community-acquired pneumonia, including nursing-home pneumonia

Mandatory

8 Nosocomial pneumonia, including ventilator-associated pneumonia

Mandatory

9 Aspiration pneumonia

Mandatory

10 Seasonal influenza

Mandatory

11 Extrapulmonary complications

Mandatory

Module 7. Other respiratory infections

1 Fungal pulmonary infections

Mandatory

2 Lung abscess

Mandatory

3 Nocardiosis

Mandatory

4 Actinomycosis

Mandatory

5 Parasitic pneumonia

Optional

6 Travel born respiratory infections

Mandatory

Module 8. Severe viral respiratory infections

1 Viruses

Mandatory

1.1 Severe acute respiratory syndrome (SARS), Middle East respiratory syndrome (MERS), H1N1, H5N1

2 Pandemics

Mandatory

2.1 Identification

2.2 Management

2.3 Public health policies

\section{Module 9. Mycobacterial disease}

1 Pulmonary tuberculosis

Mandatory

2 Extrapulmonary tuberculosis

Mandatory

3 Latent tuberculosis infection

Mandatory

4 Nontuberculous mycobacterial infections

Mandatory 
Table 1 Continued

\section{Module 10. Chronic respiratory infections in patients with respiratory disease}

1 Asthma

Mandatory

2 COPD

Mandatory

3 Bronchiectasis

Mandatory

4 Adult cystic fibrosis bronchiectasis

Mandatory

\section{Module 11. Pulmonary infections in the immunocompromised host}

1 Neutropenic patients

Mandatory

2 HIV-infected patients

Mandatory

3 Haematological disorders and malignancy

Mandatory

4 Lung and other solid organ transplant recipients

Mandatory

5 Haematopoietic cell transplant recipients

6 Secondary immunodeficiency induced by drugs and biologicals

Mandatory

7 Primary immune deficiency syndromes

Mandatory

\section{Module 12. Pleural infections}

1 Parapneumonic effusion and empyema

Mandatory

2 Pleuritis

Mandatory

2.1 tuberculosis, bacterial, etc.

\section{Module 13. Sepsis}

1 Sepsis, severe sepsis and septic shock

Mandatory

1.1 Virulence factors involved in sepsis

1.2 Early recognition and management

1.3 Additional therapies

1.4 Biomarkers

1.5 Clinical management

\section{Module 14. Prevention of respiratory infections}

1 Vaccination

1.1 Influenza vaccination

1.2 Pneumococcal vaccination

1.3 Other vaccinations

2 Other prevention measures

Optional

2.1 Smoking cessation

2.2 Specific preventive management

2.3 Prevention of community-acquired pneumonia

2.4 Dental care

2.5 Aspiration management

3 Infection control

3.1 Infection surveillance

3.2 Universal precautions

3.3 Isolation and reverse isolation, including specific microbes in cystic fibrosis and bronchiectasis (e.g. Pseudomonas)

3.4 Infectious risks to healthcare workers

3.5 Tuberculosis control and elimination, including Bacillus Calmette-Guérin (BCG) vaccine

3.6 Immunomodulants (synthetic and microbial) 


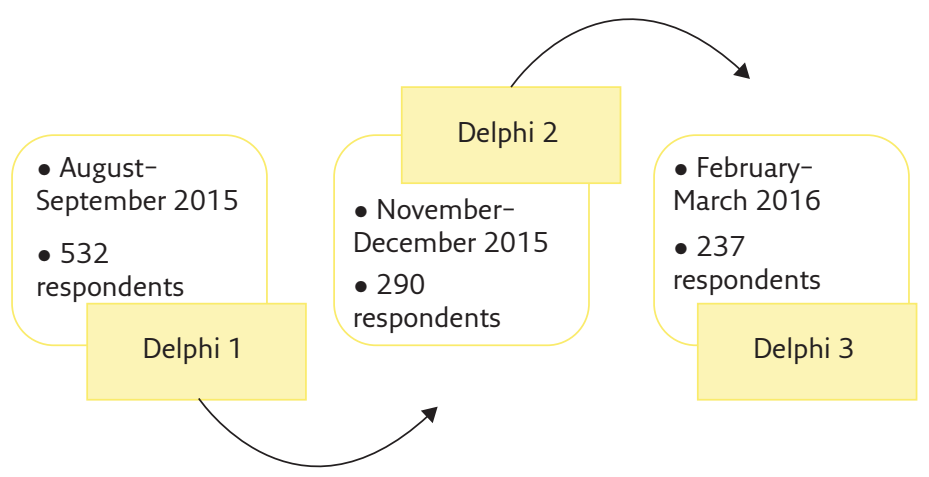

Figure 1 Overview of the Delphi process.

\section{Target audience}

As previously outlined [3], the target audience for the syllabus are qualified respiratory physicians with a special interest in respiratory infections. Based on the situational analysis and the Delphi surveys, the target audience also includes infectious diseases specialists, intensive care unit specialists, internists, trainees, researchers and microbiologists, working in public hospitals or holding an academic position.

\section{Methods}

The approach, methods, and processes used in this project have been adapted from the ERS developed educational harmonisation framework, which, to date, has been used in the development of seven postgraduate curricula (www.ersnet.org/ professional-development/ers-curriculum-designa-summary-of-projects) and four specialised skillsbased training programmes [4].

To develop the syllabus, a list of key topics was identified by the expert task force. A modified Delphi technique was applied to these topics to reach consensus. The Delphi technique is a group facilitation method that seeks to obtain consensus on the opinions of experts through a series of structured questionnaires [5]. Research and methods outlined by HEIKo [6] were used to guide the decisions taken at various stages of this study, for example survey design, undertaking data collection and analysis.

The Delphi process was phased in three rounds (figure 1). Members of ERS Assembly 10 (respiratory infections) and national experts were asked to complete an online questionnaire, which was then processed by the ERS office. The results were presented to the task force for more detailed discussion. The decisions derived from these consultations were integrated into the next Delphi round (Delphi 1). It is to be noted that although the same respondents were contacted in each round, there was a drop off in the number of responses between rounds. Respondents were asked to rate in terms of agreement whether sections should be included (mandatory or optional) or excluded. A Likert scale from 1 (strongly disagree) to 5 (strongly agree) was chosen to ensure measurement reliability over the three different Delphi rounds. Agreement was operationalised through a majority of responses in the top two points of the scale (measured as the sum of frequencies of agree and strongly agree responses). In Delphi studies consensus is considered a necessary, but not sufficient, condition for agreement concerning the inclusion of items [6]. The iteration of rounds was also required to establish the stability dimension for agreement. Stability was defined as "the consistency of responses between successive rounds of a study" [7]. Both consensus and stability dimensions were investigated in the study.

\section{Final syllabus}

The content of the syllabus was organised in 14 modules, which were considered important and necessary topics or aspects forming the basis of the respiratory infections domain (table 1).

\section{Conclusion and next steps}

The syllabus was developed to clearly define the remit for programme designers in the implementation of training and education for respiratory physicians. ERS is committed to the continuing professional development of respiratory professionals and will be using the ERS respiratory infections syllabus as a basis for several activities in the future, including:

- External courses, such as the respiratory infections course (e.g. the course held in Lisbon, Portugal, June 2018), postgraduate courses at the ERS International Congress, e-learning and other educational activities;

- Respipedia and other online resources;

- An ERS professional development programme, focusing on the eight main disease areas.

\section{Acknowledgements}

The task force would like to acknowledge Albert Osterhaus (Research Center for Emerging Infections and Zoonoses, University of Veterinary Medicine Hannover, Foundation, Hannover, Germany) for his work on this project.

The authors affiliations are as follows. Stefano Aliberti: Dept of Pathophysiology and Transplantation, University of Milano, Milan, Italy, and Respiratory Unit and Adult Cystic Fibrosis Center, Fondazione IRCCS 
Ca' Granda Ospedale Maggiore Policlinico, Milan, Italy. Amy Farr and Nathalie Tabin: European Respiratory Society, Educational Activities Dept, Lausanne, Switzerland. Francesco Blasi: Dept of Pathophysiology and Transplantation, University of Milano, Milan, Italy. Antoni Torres: Pneumology Dept, Hospital CLINIC, University of Barcelona, IDIBAPS, CIBERE, Barcelona, Spain. Mark Woodhead: Faculty of Biology, Medicine and Health, University of Manchester, Manchester, UK. Giovanni Battista Migliori: Istituti Clinici Scientifici Maugeri IRCCS, Tradate, Italy. Giovanni Sotgiu: Clinical Epidemiology and Medical Statistics Unit, Dept of Clinical and Experimental Medicine, University of Sassari, Sassari, Italy. George Dimopoulos: Dept of Critical Care, University Hospital ATTIKON, Medical School, National and Kapodistrian University of Athens, Athens, Greece. James D. Chalmers: Dept of Respiratory Medicine, Ninewells Hospital and Medical School, Dundee, UK. Felix C. Ringshausen: Dept of Respiratory Medicine, Hannover Medical School and German Center for Lung Research (DZL), Hannover, Germany. Michael R. Loebinger: Host Defence Unit, Royal Brompton Hospital, London, UK and National Heart and Lung Institute, Imperial College, London, UK. Robert Read: Academic Unit of Infection and Immunity, University of Sheffield Medical School, Sheffield, UK. Gernot Rohde: Medical clinic I, Dept of Respiratory Medicine, Goethe University Hospital, Frankfurt/Main, Germany.

\section{Conflict of interest}

S. Aliberti has nothing to disclose. A. Farr is an employee of the European Respiratory Society. N. Tabin is an employee of the European Respiratory Society. F. Blasi has nothing to disclose. A. Torres has nothing to disclose. M. Woodhead reports an honorarium for a lecture at European Pneumoupdate in 2016, 2017 and 2018. G.B. Migliori has nothing to disclose. G. Sotgiu has nothing to disclose. G. Dimopoulos has nothing to disclose. J.D. Chalmers reports grants and personal fees from Glaxosmithkline, Grifols, Boehringer-Ingelheim and Insmed, grants from Astrazeneca and Bayer Healthcare, personal fees from Aradigm, Pfizer and Napp, all outside the submitted work. F.C. Ringshausen reports grants, personal fees and other from Bayer HealthCare (research support, consulting, lecture fees and support of patient educational events), grants and personal fees from Grifols Germany (research support and consulting), grants and personal fees from Insmed Germany (research support, consulting and reimbursement for study participation/patient recruitment), personal fees from Brahms/Thermo Fisher Scientific (consulting), personal fees from Astra Zeneca (lecture fees), personal fees and other from Forest (consulting and support of patient educational events), personal fees from Cellestis/Qiagen (lecture fees), personal fees and other from Chiesi (travel reimbursement and support of patient educational events), other from Abbott (support of patient educational events), personal fees and other from Gilead (travel reimbursement and support of patient educational events), other from Pfizer (support of patient educational events), other from Oxycare (support of patient educational events), other from Novartis (support of patient educational events), other from Heinen \& Löwenstein (support of patient educational events), other from MSD (support of patient educational events), grants and other from InfectoPharm (research support and support of patient educational events), all outside the submitted work. M.R. Loebinger has nothing to disclose. R. Read has nothing to disclose. G. Rohde reports personal fees from Pfizer, Boehringer Ingelheim, Solvay, GSK, Essex Pharma, MSD, Roche and Novartis for lectures including service on speakers' bureaus outside the submitted work and/or consultancy during advisory board meeting and personal fees from GSK for travel accommodations/meeting expenses, outside the submitted work.

\section{References}

1. Global Health Estimates: Death by Cause, Age, by Country and by Region, 2000-2016. Geneva, World Health Organisation, 2018.

2. World Health Organization. World health statistics 2011. World Health Organization, 2011.

3. Niculescu A, Noel J-L, Aliberti S, et al., Introducing a new HERMES project on respiratory infections. Breathe 2016; 12: 5-7.

4. Farr A, Clementsen P, Herth F, et al. Endobronchial ultrasound: launch of an ERS structured training programme. Breathe 2016; $12: 217$
5. Yousuf MI. Using experts' opinions through Delphi technique. Pract Assess Res Eval 2007; 12: 1-8.

6. Heiko A. Consensus measurement in Delphi studies: review and implications for future quality assurance. Technol Forecast Soc Change 2012; 79: 1525-1536.

7. Dajani JS, Sincoff MZ, Talley WK. Stability and agreement criteria for the termination of Delphi studies. Technol Forecast Soc Change 1979; 13: 83-90. 